In order to obtain an optimal representation of the experimental spectra by the theoretical spectra in Figure 9, the diffusion coefficient $D$ also was taken as an adjustable parameter. Clearly, we would expect $D$ to be independent of the electrical field $E$. In Figure 11, we show the fitted diffusion coefficients $D$ as a function of the applied field, which are definitely not independent of $E$. It is interesting to note, however, that with vanishing field $E$ the diffusion coefficient extrapolates to $D=1.09 \times 10^{-8} \mathrm{~cm}^{2} \mathrm{~s}^{-1}$, a value close to $D=1.02 \times 10^{-8} \mathrm{~cm}^{2} \mathrm{~s}^{-1}$, the diffusion coefficient of particles with the hydrodynamic diameter $481 \mathrm{~nm}$. We are thus forced to conclude that an additional broadening mechanism, different from simple diffusion, is operative as the electric field is turned on. Although details of this field-dependent broadening mechanism are presently unknown, one simple possible explanation could be size and/or charge polydispersity, which would lead to a distribution of the electrophoretic mobility of the particles and thus to a field-dependent broadening of the spectra. Systematic investigations of this phenomenon are in preparation.

\section{Conclusion}

In this paper, electrophoretic light scattering in the presence of electroosmosis is investigated both theoretically and experi- mentally. For a rectangular scattering cell, we observed a wide range of inhomogeneously broadened light-scattering spectra that can be fully accounted for by the theoretical expressions derived above. For a selected colloidal suspension, it is shown that the electrokinetic light-scattering results are in quantitative agreement with results obtained by the method of microelectrophoresis.

In the early stage of this investigation, we used various types of scattering cells with more or less poorly defined electroosmotic velocity profiles. Electrophoretic velocities determined with such cells should be considered with caution since they may differ by as much as a factor of 2 from those determined under well-defined conditions.

Since the well-established method of microelectrophoresis is time consuming and tedious and cannot be applied to particles with diameters less than about $300 \mathrm{~nm}$, the extended light-scattering technique described in this paper may become a quick and reliable method in colloid and surface chemistry to determine electrokinetic parameters.

Acknowledgment. Financial support of this work by the Deutsche Forschungsgemeinschaft and by the Fonds der Chemischen Industrie is gratefully acknowledged.

\title{
Volume Correction for Bead Model Simulations of Rotational Friction Coefficients of Macromolecules
}

\author{
Jan Antosiewicz*, ${ }^{*}$ and Dietmar Porschke* \\ Max Planck Institut für Biophysikalische Chemie, 3400 Göttingen-Nikolausberg, FRG \\ (Received: November 8, 1988; In Final Form: February 23, 1989)
}

\begin{abstract}
Bead model simulations according to Kirkwood and Riseman proved to be very useful for the evaluation of hydrodynamic parameters of macromolecules, but the simulated rotational parameters are known to be associated with systematic deviations in many cases. We show that these deviations can be reduced considerably by a correction based on "solvent-accessible volumes", which are defined as the sum of the bead "cones" connected with the solvent-accessible surface. The procedure is tested by calculations of translational and rotational friction coefficients of spheres and cylinders, which are modeled by small beads touching the surface of the simulated object. The results obtained by the new procedure are in satisfactory agreement with exact analytical results. The new procedure is applied to bead model simulations of $\alpha$-chymotrypsin and tRNA ${ }^{\text {Phe; }}$ these simulations are based on the known X-ray structures and are shown to be consistent with available experimental data on translational and rotational diffusion.
\end{abstract}

\section{Introduction}

Bead models are currently used in the study of hydrodynamic properties of rigid- and flexible-chain macromolecules. ${ }^{1-3}$ In the treatment of bead models developed by Kirkwood and Riseman, ${ }^{4}$ and subsequently by others (for review, see ref 1 ), the frictional force $\vec{F}_{i}$ experienced by the $i$ th bead of a moving-bead assembly is described by the equation

$$
\vec{F}_{i}+\zeta_{i} \sum_{j \neq i}^{N} T_{i, j} \vec{F}_{j}=-\zeta_{i}\left(\vec{u}_{i}-\vec{v}_{i}^{0}\right) \quad i=1, \ldots, N
$$

where $N$ is the number of beads in the assembly; $\zeta_{i}=6 \pi \eta \sigma_{i}$ is the Stokes' law friction coefficient of a sphere of radius $\sigma_{i}$ in a solvent of viscosity $\eta ; \vec{u}_{i}$ is the velocity of the $i$ th bead; $\vec{v}_{i}{ }^{0}$ is the velocity that the solvent would have at the position of the $i$ th bead in the absence of the bead assembly; $T_{i, j}$ is the hydrodynamic interaction tensor, which describes the influence on the solvent velocity at the position of the $i$ th bead due to the presence of the $j$ th bead of the assembly. ${ }^{1,5}$

For the calculation of rotational friction coefficients, velocities $\vec{u}_{i}$ are taken as

\footnotetext{
${ }^{+}$On leave from the Department of Biophysics, Warsaw University, 02-089 Warsaw, Poland
}

$$
\vec{u}_{i}=\vec{\omega} \times \vec{r}_{i}
$$

where $\vec{r}_{i}$ is the position vector of the $i$-th bead with respect to the origin of the coordinate system and $\bar{\omega}$ is the angular velocity of the macromolecule. These velocities are used in eq 1 for evaluation of the forces, and subsequently, frictional torques are calculated as

$$
\vec{T}_{i}^{\prime}=\vec{r}_{i} \times \vec{F}_{i}
$$

As was noticed some time ago, this procedure leads to erroneous results for rotational friction coefficients. ${ }^{1,6,7}$ For example, a single bead located at the origin of the coordinate system is associated with $T_{1}{ }^{\prime}=0$ because of $r_{1}=0$, whereas the correct result is $T_{1}$ $=8 \pi \eta \sigma_{1}^{3} \omega$.

(1) Garcia de la Torre, J.; Bloomfield, V. A. Q. Rev. Biophys. 1981, 14, 81.

(2) Yamakawa, H. Modern Theory of Polymer Solutions; Harper and Row: New York, 1971 .

(3) Wegener, W. A. J. Chem. Phys. 1982, 76, 6425.

(4) Kirkwood, J. G,; Riseman, J. R. J. Chem. Phys. 1948, 16, 565.

(5) Oseen, C. W. Hydrodynamik. In Mathematik und ihre Anwendungen in Monographien und Lehrbüchern; Hilb, E., Ed.; Akad.-Verlagsges.: Leipzig, Germany, 1927

(6) Wilson, R. W.; Bloomfield, V. A. Biopolymers 1979, 18, 1205.

(7) Garcia Bernal, J. M.; Garcia de la Torre, J. Biopolymers 1981, $20,129$. 
Recently, Garcia de la Torre and Rodes ${ }^{8}$ proposed to correct the principal rotational resistance coefficients calculated according to the Kirkwood-Riseman procedure by adding to each of them the term $6 \eta V$, where $V$ is the volume of the hydrated macromolecule. This procedure leads to some overestimation of rotational resistance coefficients. Thus, translational and rotational diffusion coefficients calculated for bead models according to the available procedures are not consistent. If the bead model is exactly adapted to a known translational resistance coefficient, then the rotational resistance coefficient calculated for the same model is too low when no correction is applied and too large when corrected according to Garcia de la Torre and Rodes. Under these conditions, it is not really possible to compare experimental results for a given macromolecule obtained by different procedures, like sedimentation velocity and transient electric dichroism. Obviously, consistent procedures are required for any quantitative analysis of experimental data.

In the present contribution, we show that the results of bead model simulations are consistent when the correction presented by Garcia de la Torre and Rodes ${ }^{8}$ is modified. We start from the known fact that simulations of translational and rotational friction coefficients for a given object are more accurate when the object is modeled by an increasing number of beads of decreasing radius distributed on the surface of the object (where the frictional forces act). In the limit of an infinite number of infinitely small beads on the surface of the object, the simulation procedure is exact for translational friction coefficients ${ }^{9}$ and is expected to be exact for rotational friction coefficients - without any volume correction. Thus, the correction term for models with a limited number of beads should not be based on the total volume but should be dependent on the size of the beads. The correction should decrease when a given object is modeled in detail by an increasing number of beads with decreasing radius. For this reason, we propose a correction based on a volume, which may be called the "solvent-accessible volume", because it is calculated in close analogy to the solvent-accessible surface of macromolecules. ${ }^{10,11}$ The accuracy of the new procedure has been tested by a comparison of simulations on different models of a sphere with the analytical result. Furthermore, simulations are presented and discussed for cylinders as well as for detailed models of yeast tRNA ${ }^{\text {Phe }}$ and $\alpha$-chymotrypsin from bovine pancreas.

\section{Methods of Calculation}

2.1. Hydrodynamic Parameters for Rigid-Bead Models of Macromolecules. Translational and rotational resistance coefficients were calculated by a Fortran program written on the basis of a review article by Garcia de la Torre and Bloomfield. ${ }^{1}$ In the present context, a brief description of the main steps of the simulation should be sufficient.

By conversion of eq 1 , we obtain for the forces

$$
\vec{F}=-\boldsymbol{Z}_{\imath} \vec{u}
$$

where $\vec{F}=\sum_{i} \vec{F}_{i}$ and $\vec{u}$ is the velocity of the whole assembly relative to the solvent. This equation may be used to determine the components of the translational friction tensor $\Xi_{t}$. The rotational resistance tensor is found from the relation

$$
\vec{T}_{O}=-\bar{\Xi}_{0, \tau} \vec{\omega}
$$

where $\vec{T}_{0}=\sum_{i} \vec{T}_{i}$ and subscript $O$ indicates that the tensor and the final torque are dependent on the origin of the coordinate system. For results with physical meaning, the rotational resistance tensor has to be referred to the "center of resistance" ${ }^{12}$ This center is evaluated from the "translational-rotational resistance coupling tensor", which is defined by

$$
\vec{T}_{\mathrm{O}}=-\boldsymbol{\Xi}_{\mathrm{O}, \mathrm{c}} \overrightarrow{\mathrm{u}}
$$

The four tensors $\Xi_{1}, \Xi_{O, r}, \Xi_{O, c}$, and $\Xi_{O, c}{ }^{\mathrm{T}}$ form the $6 \times 6$ gener-

(8) Garcia de la Torre, J.; Rodes, V. J. Chem. Phys. 1983, 79, 2454

(9) Swanson, E.; Teller, D. C.; de Häen, C. J. Chem. Phys. 1978, 68, 5097

(10) Lee, B.; Richards, F. M. J.. Mol, Biol 1971, 55, 379.

(11) Matthew, J. B.; Hanania, G. I. H.; Gurd, F. R. N. Biochem. Biophys. Res. Commun. 1978, 81, 410.

(12) Brenner, H. J. Colloid Interface Sci. 1967, 23, 407.
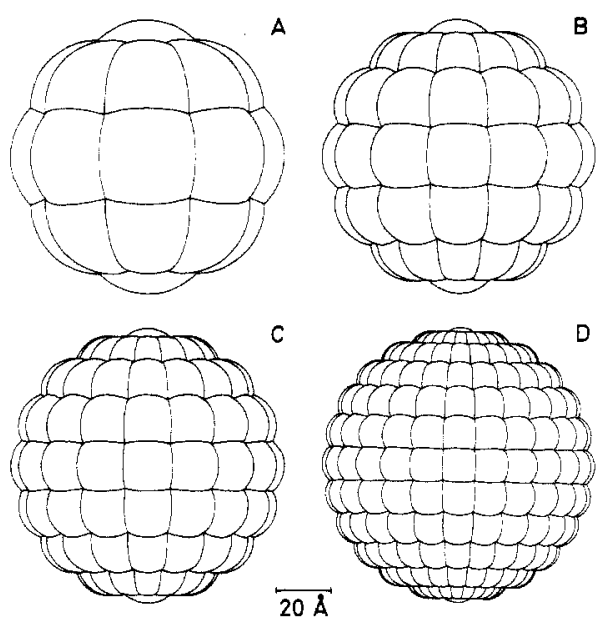

Figure 1. Four models for a $50-\AA$ sphere: (A) 26 beads with radius 25 $\AA$; (B) 62 beads with radius $15 \AA$; (C) 114 beads with radius $12 \AA$; and (D) 266 beads with radius $8 \AA$.

alized resistance tensor $\mathbf{R}^{12}$ (subscripts $t, r$, and c denote translational, rotational, and coupling tensors, respectively; superscript $T$ denotes the transposed form). We have solved the system of $N$ linear equations (1) by the DLSARG subroutine described in the IMSL Math/Library Manual. ${ }^{13}$ For nonoverlapping beads, we used the expression for the hydrodynamic interaction tensor given by Garcia de la Torre and Bloomfield, ${ }^{14}$ and for overlapping beads, that of Rotne and Prager. ${ }^{15}$ By this procedure, we obtain the rotational resistance tensor without correction terms. For corrections according to Garcia de la Torre and Rodes, ${ }^{8}$ we add $6 \eta \mathrm{V}$ to the diagonal components of this subtensor, where $V$ is the total volume of the model; according to our new procedure, we add $6 \eta V_{\mathrm{sa}}$, where $V_{\mathrm{sa}}$ is the solvent-accessible volume. Friction coefficients are converted to diffusion coefficients by the generalized Einstein relation ${ }^{17}$

$$
\mathbf{D}=k \mathrm{TR}^{-1}
$$

where $\mathbf{D}$ is the generalized $6 \times 6$ tensor of diffusion coefficients. Rotational relaxation times are calculated from rotational diffusion coefficients, referred to the "center of diffusion"18 as described by Wegener et al: ${ }^{19}$

2.2. Modeling of a Sphere by Beads. Our models for a sphere of radius $\zeta$ are constructed from a given number of small beads of radius $\sigma$, which are distributed uniformly with their centers on the surface of the sphere of radius $\rho=\zeta-\sigma$. First, we generate coordinates for spheres located in the $x z$-plane. We start from $n$ spheres distributed on the arc, which is located in the first quarter of the $x z$-plane. They have coordinates

$$
\begin{array}{cc}
x_{i}=\rho \cos a_{i} \quad y_{i}=0 & z_{i}=\rho \sin a_{i} \\
a_{i}=(i-1) \pi /(2 n) & i=1, \ldots, n
\end{array}
$$

Further, $n$ beads have coordinates

$$
\begin{gathered}
x_{j}=-x_{i} \quad y_{j}=y_{i} \quad z_{j}=z_{i} \\
j=n+1, \ldots, 2 n \quad i=1, \ldots, n
\end{gathered}
$$

And, further, $2 n-2$ beads have coordinates

$$
\begin{gathered}
x_{j}=x_{i} \quad y_{j}=y_{i} \quad z_{j}=-z_{i} \\
j=2 n+1, \ldots, 4 n-2 \quad i=2, \ldots, 2 n-1
\end{gathered}
$$

(13) IMSL-Fortran Subroutines for Mathematical Applications; IMSL: Houston, TX, 1987; Vol. 1, p 11 .

(14) Garcia de la Torre, J.; Bloomfield, V. A. Biopolymers 1977, 16, 1747.

(15) Rotne, J.; Prager, S. J. Chem. Phys. 1969, 50, 4831.

(16) Tirado, M. M.; Martinez, C. L.; Garcia de la Torre, J. J. Chem. Phys. $1984,81,2047$

(17) Brenner, H. J. Colloid Sci. 1965, 20, 104.

(18) Harvey, S. C.; Garcia de la Torre, J. Macromolecules 1980, 13, 960

(19) Wegener, W. A.; Dowben, R. M.; Koester, V. J. J. Chem. Phys. 1979, 


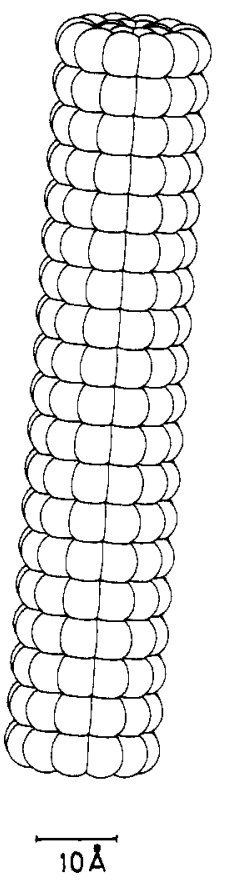

Figure 2. Bead model of a cylinder with length $96 \AA$ and diameter 20 $\AA$ (model B). Solvent-accessible volumes and volumes occupied by beads are 4000 and $10000 \AA^{3}$ for cylinder $A$ and 8300 and $20200 \AA^{3}$ for model B.

At this moment, we have the coordinates of $4 n-2$ beads. Now, we rotate these beads around the $z$-axis, subsequently by angles $a_{i}$ for $i=1, \ldots, 2 n-1$. Now, we have $2 n(4 n-2)$ beads. Finally, we add two last beads with the coordinates $(0,0, \pm \rho)$. Thus, in total, the model is composed of $N=8 n^{2}-4 n+2$ beads. Four different models, which represent a sphere with a radius $50 \AA$ and which have been constructed by the procedure described above, are shown in Figure 1.

2.3. Modeling of a Cylinder by Beads. First, we generate a set of $4 n$ beads with radius $\sigma$, arranged with their centers in the $x y$-plane on a circle of radius $\rho=\zeta-\sigma$. The procedure is as for the first $4 n-2$ beads for the models of spheres; two beads are added on the $y$-axes with the $y$-coordinate $\pm \rho$. In the first ring, the $z$-coordinates of all beads are zero. For additions of more rings, we translate the first one to coordinates $z= \pm(5 n \sigma / 3)$ with $n=$ $1, \ldots, N$. The two ends of the cylinder are closed by a set of appropriate rings formed by beads of the same size. The final bead assembly is considered as a model for a cylinder with diameter $d=2 \zeta$ and length $L=10 N \sigma / 3+2 \sigma$. Two cylinders were modeled by $3-\AA$ beads: (A) $L=46 \AA, d=20 \AA, 124$ beads; (B) $L=96 \AA, d=20 \AA, 244$ beads. Model B is presented in Figure 2.

2.4. Calculation of Solvent-Accessible Volume. Our program for calculations of solvent-accessible volumes of macromolecules was developed in analogy to the procedure described by Lee and Richards. " A given bead model is sliced by a series of parallel planes (e.g., perpendicular to the $z$-axis of the coordinate system), with a spacing $\Delta$. The intersection of each bead with a given plane appears as a circle. Because some of the beads overlap with each other, for each circle only some part of its contour is free, the rest being covered by other circles. The free arc has contact with the surrounding solvent and contributes to the solvent-accessible surface of the bead, and thus, the corresponding sector is used for the calculation of the solvent-accessible volume. Figure 3a shows one of the models presented in Figure 1 sliced by three planes, with one of the beads being dashed. Figure $3 \mathrm{~b}$ shows the result of intersection of plane 1 with the model, including the free arc of the circle belonging to the dashed bead. By multiplication of the circular sector area (dashed in Figure $3 \mathrm{~b}$ ) with the interplanar spacing $\Delta$, we get the contribution to the solvent-accessible volume of the dashed bead from a given plane. Summation over all beads and layers provides the solvent-accessible volume of the

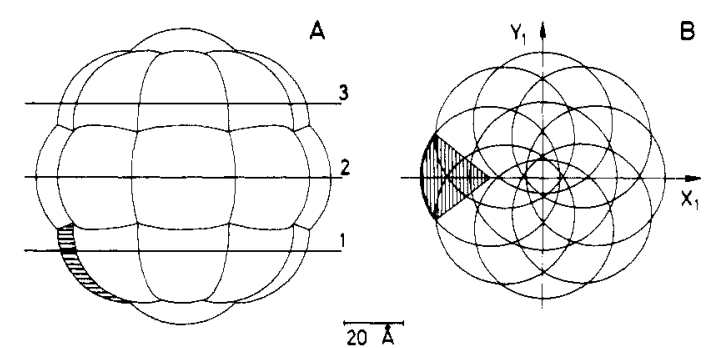

Figure 3. Definition of solvent-accessible volumes. (A) The model sliced by three parallel planes. One bead is dashed. (B) Circles arising from intersection of the model with plane 1 . Free arc and the area for calculation of the contribution to solvent-accessible volume for the dashed bead are shown.

model. Obviously, the spacing $\Delta$ has to be sufficiently small.

When a given bead model has some internal cavities, as in models B-D, Figure 1, we have introduced some additional beads, which cover the internal surfaces of beads, that have no contact with the solvent. These beads simply serve for the correct calculation of solvent-accessible volumes, which should not include contributions from internal surfaces. In the case of models shown in Figure 1B-D, we introduced one additional bead centered at the origin of the coordinate system, with radius $\zeta-\sigma$ (model $A$ does not have an internal cavity, because it has been constructed from large beads). Obviously, such additional beads do not contribute to the solvent-accessible volume.

In the case of cylinders, the internal volume is filled with beads of radius $\zeta-\sigma$, which are aligned on the $z$-axes with coordinates $z_{i}^{ \pm}= \pm i(\zeta-\sigma)$.

Our program also calculates solvent-accessible surfaces, as defined in ref 10 . The program was checked extensively on a set of test data, as, e.g., one shown in Figure 3a, for which free arcs of the circles may be easily determined.

2.5. Calculations of Volumes of Bead Models. As mentioned above, Garcia de la Torre and Rodes ${ }^{8}$ proposed to take the volume of the hydrated macromolecule for correction of the diagonal components of the rotational resistance tensor. In the present calculations, we take the volume of the bead model for this purpose. This volume is evaluated without contributions from internal spaces, which are not occupied by beads. Obviously, the correction terms would be still larger, if based on the total volume including the internal spaces.

Because it is not easy to calculate the volume occupied by overlapping beads analytically, it was calculated by a Monte Carlo method. According to this method, the model is placed in a box of defined dimensions and a set of random points is generated inside this box. The volume of the model is given by the fraction of random points inside the model multiplied by the volume of the box.

2.6. Reference Values of Friction and Diffusion Coefficients. Translational and rotational resistance coefficients of a sphere of radius $\zeta$ in a solvent with viscosity $\eta$ are given by Stokes' equations

$$
\begin{gathered}
f_{\mathrm{t}, \mathrm{sphe}}=6 \pi \eta \zeta \\
f_{\tau, \text { sphe }}=8 \pi \eta \zeta^{3}
\end{gathered}
$$

For a sphere of $50 \AA$ in water of $20^{\circ} \mathrm{C}$ (viscosity $0.01002 \mathrm{P}$ ), the translational resistance coefficient is $9.44 \times 10^{-8} \mathrm{~g} / \mathrm{s}$ and the rotational resistance coefficient is $3.15 \times 10^{-20} \mathrm{~cm}^{2} \mathrm{~g} / \mathrm{s}$.

We have calculated the corresponding parameters for our cylinders $\mathrm{A}$ and $\mathrm{B}$ according to Tirado et al. ${ }^{16}$ Under the conditions defined above, the translational diffusion coefficients are 12.5 and $8.22 \times 10^{-7} \mathrm{~cm}^{2} / \mathrm{s}$ for sidewise motion and 14.3 and 10.4 $\times 10^{-7} \mathrm{~cm}^{2} / \mathrm{s}$ for lengthwise motion; the rotational diffusion coefficients for reorientation of the main axes are 22.2 and 4.77 $\times 10^{6} \mathrm{~s}^{-1}$ (the first value refers to cylinder $\mathrm{A}$ and the second to $B)$. These values serve as the basis for comparison with the results of our bead-model simulations. 
TABLE I: Total Volumes of the Models, Volumes Occupied by Beads, Solvent-Accessible Surfaces, and Solvent-Accessible Volumes for Four Models of a 50- $\AA$ Sphere Presented in Figure $1^{a}$

\begin{tabular}{ccccc}
\hline $\begin{array}{c}\text { type of } \\
\text { model }\end{array}$ & $\begin{array}{c}\text { total vol of } \\
\text { model, } \AA^{3}\end{array}$ & $\begin{array}{c}\text { vol occupied } \\
\text { by beads, } \AA^{3}\end{array}$ & $\begin{array}{c}\text { solvent- } \\
\text { accessible } \\
\text { surface, } \AA^{2}\end{array}$ & $\begin{array}{c}\text { solvent- } \\
\text { accessible } \\
\text { vol, } \AA^{3}\end{array}$ \\
\hline A & 465000 & 465000 & 30200 & 253000 \\
B & 460000 & 415000 & 32000 & 157000 \\
C & 467000 & 385000 & 32500 & 127000 \\
$D$ & 486000 & 303000 & 35800 & 92700
\end{tabular}

a For comparison, surface of the sphere is $31415.9 \AA^{2}$ and its volume is $523599 \AA^{3}$. Volumes of the models and volumes occupied by beads are calculated three times with 10000 random hits each time. Volumes of the models were calculated with additional internal beads filling the empty space of a given model, if necessary. Presented values are the means of these three results. Standard deviations are always smaller than $1.5 \%$ of the mean value. Spacing of the planes for calculation of solvent-accessible surfaces and volumes was $0.1 \AA$.

TABLE II: Translational and Rotational Resistance Coefficients for Four Models of a 50- $\AA$ Sphere, Shown in Figure $1^{a}$

\begin{tabular}{|c|c|c|c|c|}
\hline \multirow[b]{2}{*}{$\begin{array}{r}\text { type of } \\
\text { model }\end{array}$} & \multirow{2}{*}{$\begin{array}{l}\text { translational } \\
\text { resistance } \\
\text { coeff, } 10^{-8} \mathrm{~g} / \mathrm{s}\end{array}$} & \multicolumn{3}{|c|}{ rotational resistance coeff, $10^{-20} \mathrm{~cm}^{2} \mathrm{~g} / \mathrm{s}$} \\
\hline & & $\begin{array}{l}\text { without } \\
\text { correction }\end{array}$ & & \\
\hline A & & & & \\
\hline B & & & & \\
\hline C & & & & \\
\hline D & $8.59(-9.0)$ & $2.29(-27.3)$ & $4.11(+30.5)$ & $2.85(-9.5)$ \\
\hline
\end{tabular}

${ }^{a}$ In parentheses, percent deviations from analytical results are given. Data referred to $20.0^{\circ} \mathrm{C}$.

TABLE III: Translational and Rotational Diffusion Coefficients for Models of Two Cylinders with Parameters (A) $L=46 \AA, d=20 \AA$, 124 Beads; (B) $L=96 \AA, d=20 \AA, 244$ Beads $^{a}$

\begin{tabular}{|c|c|c|c|c|}
\hline \multirow[b]{2}{*}{$\begin{array}{l}\text { type of } \\
\text { model }\end{array}$} & \multicolumn{2}{|c|}{$\begin{array}{l}\text { translational diffusion } \\
\text { coeff, } 10^{-7} \mathrm{~cm}^{2} / \mathrm{s}\end{array}$} & \multicolumn{2}{|c|}{$\begin{array}{l}\text { rotational diffusion coeff, } \\
110^{6} \mathrm{~s}^{-1}\end{array}$} \\
\hline & $\begin{array}{l}\text { sidewise } \\
\text { motion }\end{array}$ & $\begin{array}{c}\text { lengthwise } \\
\text { motion }\end{array}$ & $\begin{array}{l}\text { corrected } \\
\text { with } V\end{array}$ & $\begin{array}{l}\text { corrected } \\
\text { with } V_{\mathrm{sa}}\end{array}$ \\
\hline $\begin{array}{l}\text { A } \\
\text { B }\end{array}$ & $\begin{array}{c}13.3(+6.4) \\
8.56(+4.1)\end{array}$ & $\begin{array}{l}15.5(+8.4) \\
11.2(+7.7)\end{array}$ & $\begin{array}{r}19.8(-10.8) \\
4.71(-1.3)\end{array}$ & $\begin{array}{c}24.0(+8.1) \\
5.14(+7.8)\end{array}$ \\
\hline
\end{tabular}

${ }^{a}$ Radius of beads is $3 \AA$. Data refer to $20^{\circ} \mathrm{C}$ in water. In parentheses, percent deviations from results of Tirado et al. ${ }^{16}$ are given.

\section{Results and Discussion}

As shown in Table I, the solvent-accessible volume of bead models used for the new correction procedure is clearly smaller than the volume occupied by beads of the models, which has been applied for corrections according to Garcia de la Torre and Rodes. The translational and rotational friction coefficients resulting from the different procedures for various sphere models are compiled in Table II. In all cases, the rotational friction coefficients obtained by the new procedure are closer to the analytical results than those calculated by the other procedure. For model A, which has been constructed from relatively large beads, the translational friction coefficient is too small by $15 \%$, whereas the rotational friction coefficient is too large by $10 \%$. These deviations in opposite directions are avoided by models constructed from smaller beads. In this respect, the friction coefficients for models B-D obtained according to the new procedure are consistent in the sense that the deviations for translation and rotation are in the same direction. In general, the deviations decrease with decreasing radius of the beads used for construction of the model. In the case of models B-D, the volume of an individual bead is $2.7,1.3$, and $0.5 \%$ of the volume of the modeled sphere, respectively. It may be suspected that particularly small beads cannot be used for simulations assuming continuum fluid mechanics. For the present applications, our approach can be justified by the fact that our beads are large and our complete models are very large compared to the solvent molecules (for a recent discussion of

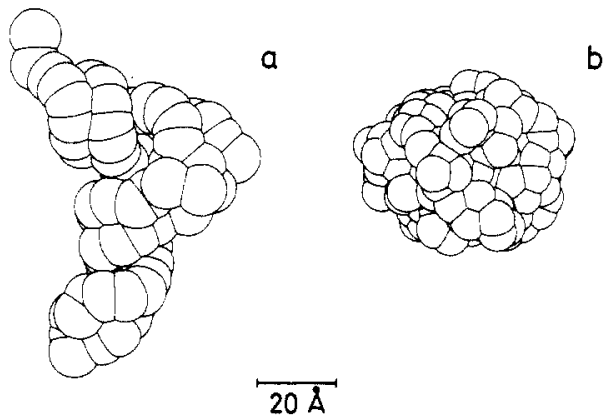

Figure 4. Bead models of yeast tRNA ${ }^{\text {Phe }}$ (a) and $\alpha$-chymotrypsin (b; monomer $\mathrm{A}$ of the dimer found in the crystal structure). Calculated solvent-accessible volumes of models are $16000 \AA^{3}$ for $\alpha$-chymotrypsin and $26000 \AA^{3}$ for tRNA.

TABLE IV: Translational Diffusion Coefficients and Rotational Relaxation Times for Models of Yeast tRNA ${ }^{\text {Phe }}$ and $\alpha$-Chymotrypsin Molecules Shown in Figure 3 (Data Referred to $20.0^{\circ} \mathrm{C}$ )

\begin{tabular}{|c|c|c|c|c|}
\hline \multirow[b]{2}{*}{ molecule } & \multicolumn{2}{|c|}{$\begin{array}{l}\text { translational diffusn } \\
\text { coeff, } 10^{-7} \mathrm{~cm}^{2} / \mathrm{s}\end{array}$} & \multicolumn{2}{|c|}{$\begin{array}{l}\text { rotational relaxn } \\
\text { times, ns }\end{array}$} \\
\hline & calcd & exptl & calcd & $\operatorname{expt}^{a}$ \\
\hline chymotrypsin & $\begin{array}{l}9.28 \\
9.29^{c}\end{array}$ & $10.2^{23}$ & $\begin{array}{l}30.3^{b} \\
30.2^{b, c}\end{array}$ & $30.6^{b}$ \\
\hline tRNA & 8.15 & $7.2-8.2^{d 24}$ & 23.5 & 23.5 \\
\hline
\end{tabular}

${ }^{a}$ Our data to be published. ${ }^{b}$ Referred to $2.0^{\circ} \mathrm{C}$. ${ }^{c} 37$ internal beads with no contact with the solvent have been removed. ${ }^{d}$ Depending on salt conditions and concentration.

"atomic" friction coefficients see ref 20).

The results obtained for cylinders, which are compiled in Table III, lead to the same general conclusion as given above. Since analytical results are not available for cylinders, we use the parameters given by Tirado et al. for comparison. Compared to this standard, the friction coefficients calculated according to the new procedure appear to be more accurate. Moreover, these friction coefficients are consistent in the sense described above.

Because it is virtually impossible to represent a given object in every detail by a limited number of beads, it has been suggested to compensate for differences of the model and the object by adjusting the size of the model such that its total volume is equivalent to that of the object. In the present case, it is very simple to calculate the analytical friction coefficients for spheres with volumes equivalent to the total volume of the bead models $\left(9.08,9.05,9.08,9.22 \times 10^{-8} \mathrm{~g} / \mathrm{s}\right.$ for translation and $2.80,2.77$, $2.80,2.93 \times 10^{-20} \mathrm{~cm}^{2} \mathrm{~g} / \mathrm{s}$ for rotation - for models A-D, respectively). These values demonstrate that friction coefficients simulated according to the new correction procedure for bead models with equivalent volumes are clearly more accurate than those obtained by other procedures.

Finally, the new correction procedure has been applied to simulations of two macromolecules of known crystal structure. We use $\alpha$-chymotrypsin determined by Tsukada and Blow ${ }^{21}$ and tRNA ${ }^{\text {Phe }}$ determined by Ladner et al. ${ }^{22}$ as examples and simply substitute one bead for each amino acid or nucleotide, respectively. According to their average dimensions, the bead radii should be in the range 5-7 $\AA$. The exact bead radii for $\alpha$-chymotrypsin and for tRNA ${ }^{\text {Phe }}$ have been adjusted to 5.1 and $6.6 \AA$, because these values lead to a satisfactory agreement of simulated dichroism decay time constants with experimental ones. The models obtained by this approach are shown in Figure 4, and the corresponding set of simulated hydrodynamic parameters are presented in Table

(20) Pastor, R. W. Karplus, M. J. Phys. Chem. 1988, 92, 2636.

(21) Tsukada, H.; Blow, D. M. J. Mol. Biol. 1985, 184, 703.

(22) Ladner, J. E.; Jack, A.; Robertus, J. D.; Brown, R. S.; Rhodes, D.; Clark, B. F. C.; Klug, A. Nucleic Acids Res. 1975, 2, 1629.

(23) Schwert, G. W.; Kaufman, S. J. Biol. Chem. 1951, 190, 807

(24) Olson, T.; Fournier, M. J.; Langley, K. H.; Ford, N. C., Jr. J. Mol Biol. 1976, 102, 193. 
IV together with experimental values of translational diffusion coefficients and rotational relaxation time constants. Since the details of the hydrated structures of these -macromolecules in solution are not known, the simulated data cannot be used for an absolute test of our procedure. Nevertheless, a relative test is possible by a comparison of data for rotational and translational diffusion. As shown in Table IV, the experimental data for both modes of diffusion can be represented by one bead model each for $\alpha$-chymotrypsin and for tRNA ${ }^{\text {Phe }}$ when we use our new procedure. Hydrodynamic simulations according to the other procedures may be used for a satisfactory fit of one mode of diffusion by a given bead model but not of both modes. We should add that the algorithm introduced for our new correction procedure does not lead to any noticeable increase in the computing time.
In summary, we may conclude that the results of hydrodynamic simulations are improved by corrections with surface-accessible volumes, because rotational friction coefficients can be predicted at a higher accuracy and also are more consistent with translational coefficients. More experimental data for translational and rotational properties of macromolecules, referred to the same buffer conditions, would be useful as a further control of the new correction procedure.

Acknowledgment. All calculations were performed at the facilities of the Gesellschaft für wissenschaftliche Datenverarbeitung $\mathrm{mbH}$, Göttingen. Models were drawn by the SHAKAL program written by E. Kellar.

Registry No. Chymotrypsin, 9004-07-3.

\title{
Study on Polymerization Mechanism of Pentacosadiynoic Acid Langmuir-Blodgett Films Using High Energy Beam Irradiations
}

\author{
Kazufumi Ogawa \\ Semiconductor Research Center, Matsushita Electric Industrial Company, Ltd., 3-15, Yagumo-Nakamachi, \\ Moriguchi, Osaka, 570 Japan (Received: October 3, 1988)
}

\begin{abstract}
Studies have been carried out on $\mathrm{X}$-ray or $\mathrm{KrF}$ excimer laser light induced polymerization of pentacosadiynoic acid (PDA) Langmuir-Blodgett (LB) films in relation to the molecular density of the films. The polymerization sensitivity of PDA LB film was affected by the arrangement or molecular density in PDA LB film. On the low-density film (A type), the polymerization occurred by irradiation with either X-ray or excimer laser light, but on the high-density film (B type), the polymerization occurred only when the irradiation was carried out with X-ray. Decomposition of polymerized films was observed by excessive irradiation by X-ray or excimer laser light on A-type film but not on B-type films. It was revealed by the spectroscopic analysis that the polydiacetylene-type polymer was obtained on the A-type films by the X-ray or the excimer laser light irradiation and that the polymerized A-type film was decomposed at the polydiacetylene bond when the irradiation continued further. It was also shown that the polyacetylene-type polymer was obtained only when the B-type film was irradiated with X-ray.
\end{abstract}

\section{Introduction}

When diacetylene derivatives are irradiated by UV light in the solid phase, they react to give polymers having conjugated double and triple bonds. ${ }^{1-4}$ Since the polymer main chain consists of a planar chain with alternating conjugated double and triple bonds that exhibit a number of interesting features with regard to optical and electronic properties such as a nonlinear optical effect or an electric conduction property, these polymers are studied widely as optical or electrofunctional materials. ${ }^{5-8}$

It was reported that, with diacetylene derivatives that have a hydrophilic group and a hydrophobic group in a molecule, ultrathin films can be prepared by the Langmuir-Blodgett (LB) method.,10 A characteristic of the LB method is that it is possible to prepare thin films having a desired molecular arrangement by building up monolayers under a properly selected surface pressure. Thus, in recent years, the LB method is thought to be one of the im-

(1) Wegner, G. Z. Naturforsch. 1969, B24, 824.

(2) Wegner, G. Makromol. Chem. 1972, 154, 35.

(3) Baughman, R. H. J. Appl. Phys. 1972, 43, 4362

(4) Wegner, G.; Schermann, W. Colloid Polym. Sci. 1974, 252, 655

(5) Wilson, E. G. J. Phys. C 1975, 8, 727.

(6) Bloor, D.; Ando, D. J.; Preston, F. H.; Stevens, G. C. Chem. Phys. Lett.

1974, 24, 407

(7) Bloor, D.; Chance, R. R. Polydiacetylenes; Nijhoff: Boston, MA, 1985.

(8) Mehring, H.; Roth, S. Electronic Properties of Polymers and Related Compounds; Springer-Verlag:" Berlin, Heidelberg, 1985.

(9) Blodgett, K. B. J. Am. Chem. Soc. 1935, 57, 1007

(10) Blodgett, K. B.; Langmuir, I. Phys. Rev. 1937, 51, 964 portant means for construction of a molecular device ${ }^{11}$ having some functions at the molecular level.

Many studies have been carried out on the polymerization process of LB films of diacetylene derivatives, ${ }^{12-14}$ and recently, it was reported that the photoreactivity of diacetylene derivatives is affected strongly by the arrangement of the diacetylene group. The photoreactivity of diacetylene derivatives whose substituents were replaced with other groups that affect the molecular arrangement in the LB films has also been studied. ${ }^{15,16}$

One of the interests in conducting these studies is to find the use of the LB layers that change their color by the action of heat, pressure, or light for application in optical recording media. ${ }^{17}$

The present study has been carried out to solve these problems and to obtain information that will be helpful for future studies to prepare functional polymer LB films from pentacosadiynoic acid (PDA: $\mathrm{CH}_{3}\left(\mathrm{CH}_{2}\right)_{11} \mathrm{C} \equiv \mathrm{CC} \equiv \mathrm{C}\left(\mathrm{CH}_{2}\right)_{8} \mathrm{COOH}$ ) LB films.

(11) Mcalear, J. H.; Wehrung, J. M. Digest of Technical Papers; 198 I, Symposium on VLSI Technology; IEEE, Electron Devices Society, Piscataway, NJ, $1981 ;$ p 82.

(12) Bubeck, G.; Tieke, B.; Wegner, G. Ber. Bunsen-Ges. Phys. Chem. 1982, 86, 495 109

(13) Bubeck, C.; Tieke, B.; Wegner, G. Mol. Cryst. Liq. Cryst. 1983, 96,

(14) Tieke, B. Lieser, G.; Weiss, K. Thin Solid Films 1983, 99, 95.

(15) Wegner, G. J. Polymn. Sci., Part B 1971, 9, 133.

(16) Nakanishi, H.; Matsuda, H.; Kato, K.; Thocharis, C.; Jones, W. Polym. Prepr. Jpn. 1984, 33, 2491.

(17) Sandaman, D. J.; Tripathy, S. K.; Elman, B. S.; Samuelson, L. A Synth. Met. 1986, 15, 229. 